\title{
Multisystemic Inflammatory Syndrome in Children Associated With SARS-CoV-2 Infection: A Case Series Report in a Pediatric Center in Mexico
}

\author{
Miguel Garcia-Dominguez ${ }^{\mathrm{a}, \mathrm{f}}$, Yanin Angeles-Meneses ${ }^{\mathrm{b}}$, Angelica Lares-Payan ${ }^{\mathrm{b}}$, \\ Carlos Alberto Velazquez-Rios ${ }^{\mathrm{c}}$, Edgardo Tostado Morales ${ }^{\mathrm{d}}$, \\ Giordano Perez-Gaxiola ${ }^{\mathrm{e}}$
}

\begin{abstract}
We report four cases of patients with multisystem inflammatory syndrome in children (MIS-C) associated with severe acute respiratory syndrome coronavirus 2 (SARS-CoV-2) infection, of which three patients presented characteristics of Kawasaki disease (KD). All presented fever of more than 3 days, and gastrointestinal involvement, significant increase in C-reactive protein (CRP), polymorphonuclear cells, procalcitonin, D-dimer, fibrinogen and troponin, lymphopenia and hypoalbuminemia. Myocardial involvement was observed in two patients. All were treated with fluids resuscitation and vasoactive therapy, $75 \%$ received intravenous immunoglobulin (IVIG) and systemic steroids. Two patients developed a transient acute kidney injury, one patient presented as acute appendicitis and developed a bilateral pleural effusion. One patient required a second dose of IVIG and boluses of methylprednisolone. None required mechanical ventilation and there were no deaths.
\end{abstract}

Keywords: SARS-CoV-2; COVID-19; Multisystem inflammatory syndrome in children; Kawasaki disease; Kawasaki disease shock syndrome; Myocarditis

\section{Introduction}

The emergence of a new respiratory virus, from the beta-coro-

Manuscript submitted September 14, 2020, accepted September 19, 2020

Published online October 21, 2020

aDepartment of Clinical Immunology, Hospital Pediatrico de Sinaloa, Culiacan, Mexico

bDepartment of Pediatric, Hospital Pediatrico de Sinaloa, Culiacan, Mexico

'Department of Infectology, Hospital Pediatrico de Sinaloa, Culiacan, Sinaloa, Mexico

${ }^{\mathrm{d}}$ Department of Emergency, Hospital Pediatrico de Sinaloa, Culiacan, Mexico eEvidence-Based Medicine Department, Hospital Pediatrico de Sinaloa, $\mathrm{Cu}-$ liacan, Mexico

${ }^{f}$ Corresponding Author: Miguel Garcia-Dominguez, Department of Clinical Immunology, Hospital Pediatrico de Sinaloa, Culiacan, Mexico.

Email: miguelgarcia.alergia@gmail.com

doi: https://doi.org/10.14740/jmc3584 navirus family called severe acute respiratory syndrome coronavirus 2 (SARS-CoV-2) in Wuhan, China in December 2019 [1], was associated with severe acute respiratory syndrome in people over 65 years with hypertension, diabetes, obesity and smoking. In the pediatric population, China reported $2.2 \%$ of cases, of which more than $90 \%$ had asymptomatic or mild and moderate symptoms. There are currently more than 27 million confirmed cases worldwide, the pediatric population is between $0.8 \%$ and $4 \%$, in Mexico it has $3.6 \%$ under 19 years of age [1-3].

At the end of April 2020, it was reported in the UK that eight pediatric patients with shock fever greater than 3 days with cardiovascular shock and hyperinflammation; later in the province of Bergamo, Italy a condition called Kawasakiinduced hyperinflammatory syndrome was reported. SARS$\mathrm{CoV}-2$ by sharing clinical and mucocutaneous characteristics of Kawasaki disease (KD) and that several series of cases have subsequently been described, considering it a serious condition that warrants intensive therapy due to shock, organ and myocardial dysfunction, as well as coronary abnormalities $[4,5]$.

\section{Case Report}

We report a series of four cases of patient with multisystem inflammatory syndrome in children (MIS-C) associated a SARS-CoV-2 infection admitted to intensive care unit (ICU) (Supplementary Material 1, www.journalmc.org).

Three girls and one boy, ages 4 to 16 , had a fever 3 to 5 days before diagnosis, a history of contact with a positive case of coronavirus disease 2019 (COVID-19) in one patient, suspected case in two patients, and no evidence of contact in one patient 4 weeks before diagnosis. SARS-CoV-2 was confirmed in all cases, one patient with nasopharyngeal reverse transcription polymerase chain reaction (RT-PCR) and three patients with positive immunoglobulin $\mathrm{G}(\operatorname{IgG})$ and negative IgM serology for SARS-CoV-2.

None of the patients had respiratory symptoms of COVID-19. All presented general malaise, asthenia, and adynamia; and $\mathrm{KD}$ was determined in three cases (two with complete $\mathrm{KD}$ and one incomplete KD). All had gastrointestinal involvement (vomiting, diarrhea and/or abdominal pain). One patient 
required an emergency operation for suspected appendicitis, which was finally diagnosed as mesenteric adenitis. The echocardiogram showed myocardial involvement in two cases, but did not find coronary abnormalities.

A significant increase in C-reactive protein (CRP), polymorphonuclear cells, procalcitonin, D-dimer and fibrinogen was observed, as well as lymphopenia and hypoalbuminemia.

All patients received fluid resuscitation and vasoactive therapy (epinephrine or norepinephrine) for at least $24-48 \mathrm{~h}$ due to hypotension; all received broad-spectrum antibiotics, without isolation of germ in blood cultures. Three patients received intravenous immunoglobulin (IVIG) $(2 \mathrm{~g} / \mathrm{kg} /$ dose $)$ and three boluses of methylprednisolone (10 - $30 \mathrm{mg} / \mathrm{kg} / \mathrm{dose})$.

Two patients developed acute kidney injury with recovery of kidney function $72 \mathrm{~h}$ after IVIG and methylprednisolone treatment. The patient who presented with acute appendicitis had an insidious evolution with ascites, bilateral pleural effusion, mild pericardial effusion, pulmonary arterial hypertension and severe tricuspid regurgitation, with persistence of fever at $36 \mathrm{~h}$, elevated leukocytosis, CRP and D-dimer. A second dose of IVIG was administered, as well as methylprednisolone boluses, enoxaparin, and bilateral intercostal drainage, with recovery 3 weeks after admission. None required mechanical ventilation and there were no deaths. Everyone is at home without medication and without complications.

\section{Discussion}

SARS-CoV-2 infection in pediatric age has been reported around $1-4.3 \%$, generally asymptomatic course to mild forms. At the end of April 2020 the first cases of a new entity called MIS-C with clinical findings similar to KD, KD shock syndrome (KDSS) and toxic shock syndrome was reported [4]. On May 1, 2020, the Royal College of Paediatrics and Child Health (RCPCH) published a case definition and guidance related to this multisystem illness, defining it as a child presenting with persistent fever, inflammation (neutrophilia, elevated CRP, and lymphopenia) and evidence of single or multiorgan dysfunction (shock, cardiac, respiratory, kidney, gastrointestinal, or neurological disorder) with additional features. This may include children fulfilling full or partial criteria for KD; exclusion of any other microbial cause, including bacterial sepsis, staphylococcal or streptococcal shock syndromes, infections associated with myocarditis such as enterovirus, and SARS-CoV-2 PCR test results may be positive or negative [4-8].

Cases have increased from tens to hundreds in several countries, with different series similarities with a greater number of cases (Table 1 [9-13]): median age between 7.5 and 11 years, male predominance from $51 \%$ to $66 \%$, confirmation of SARS-CoV-2 by nasopharyngeal RT-PCR and/or IgG/IgM serology in 65-90\%, gastrointestinal involvement (vomiting, diarrhea, or abdominal pain) in more than $80 \%$, shock in 50 $90 \%$, and myocardial dysfunction in one to two thirds of patients. Complete, incomplete KD or KDSS occurred in half of the cases due to the presence of bilateral conjunctivitis, oral changes, skin rash, and edema of the hands and feet. Laboratory findings are consistent with lymphopenia and hypoal- buminemia, marked elevation of CRP, procalcitonin, ferritin, fibrinogen, D-dimer, troponin I, and N-terminal pro-brain-type natriuretic peptide (NT-proBNP). Coronary artery abnormalities occur in one third of cases, while the development of coronary aneurysms ranges from $8 \%$ to $23 \%$. The initial treatment was IVIG $(2 \mathrm{~g} / \mathrm{kg})$ in $70-100 \%$, a second dose of IVIG up to $20 \%$, systemic steroids $(2-10 \mathrm{mg} / \mathrm{kg} / \mathrm{day})$ in $34-73 \%$ and biological therapy (anti interleukin-1 (IL-1) or IL-6) in $8-20 \%$; and liquid resuscitation, vasoactive and inotropic agents in 40$80 \%$. Mortality is less than $2.5 \%$ [9-13].

The effort to understand the immunopathology of the SARS-CoV-2 infection since the beginning of the pandemic and to establish the appropriate treatment, aimed at avoiding the serious forms and complications described under a constellation of symptoms and biochemical findings of a so-called "torm of cytokines", which is characterized by hyperinflammation that generates multiorgan and myocardial dysfunction, due to the excessive release of cytokines (IL-1 $\beta$, IL-6, IL- 8 and interferon gamma (INF $\gamma)$ ) secondary to uncontrolled immune activation, commonly observed in autoimmune diseases such as juvenile idiopathic arthritis systemic, Still's disease, systemic lupus erythematosus, as well as macrophage activation syndrome, evidenced by cytopenias, coagulopathy, hepatitis, disseminated intravascular coagulation, and hyperferritinemia [14].

MIS-C is considered to be caused by a late response to SARS-CoV-2 infection, as some patients have a negative RT-PCR but a positive $\mathrm{IgM} / \mathrm{IgG}$ serology, highlighting the involvement of aberrant innate immunity as the main mechanism. There is also evidence that antibodies to SARS-CoV-2 accentuate the disease through a facilitating mechanism that enhances viral entry or antibody replication as has been observed in dengue [15].

Three disease patterns are recognized in children hospitalized for MIS-C. The first group, with persistent fever and high levels of inflammation markers, but without characteristics of $\mathrm{KD}$, shock or organ dysfunction; the second group, with criteria for $\mathrm{KD}$; and the third group, with shock and clinical, echocardiographic and laboratory evidence of myocardial injury [12]. Three of the cases we present have characteristics of KD with abdominal pain and shock. All responded adequately to forced resuscitation with fluids, IVIG, steroids, and vasopressors.

\section{Conclusions}

Various pediatric centers are recognizing the various MIS-C phenotypes around the world, with identification of risk factors, pathogenesis, clinical course, and treatment for MIS-C. These are the first cases we identified in our pediatric care center, which posed a challenge in diagnosis and treatment. Better understanding of MIS-C will allow us to recognize this serious and potentially fatal entity.

\section{Supplementary Material}

Suppl 1. Clinical characteristics, laboratory, echocardiogram, treatment and outcomes of the four patients with COVID- 


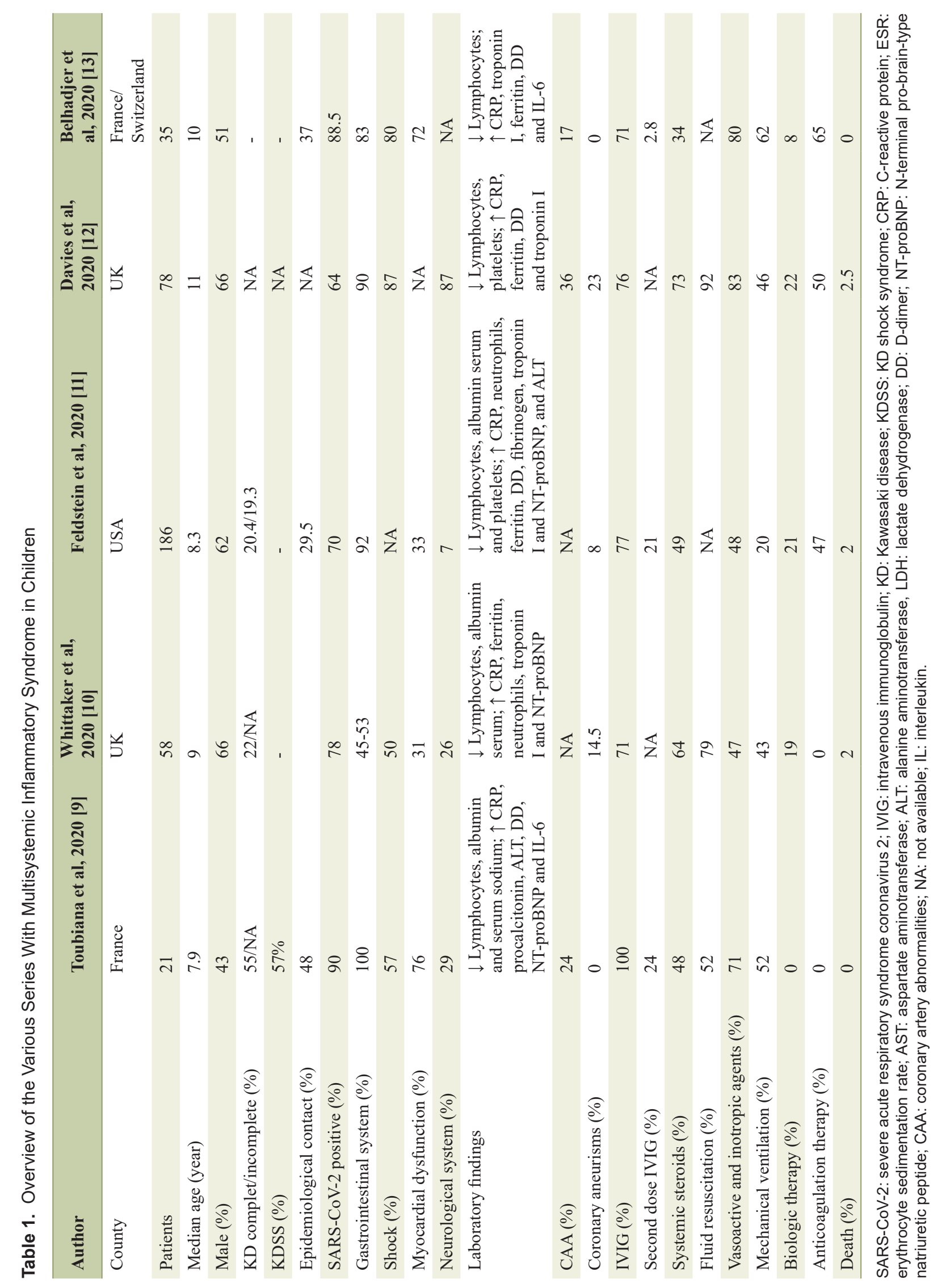


19-related multisystem inflammatory syndrome in children.

\section{Acknowledgments}

None to declare.

\section{Financial Disclosure}

None to declare.

\section{Conflict of Interest}

None to declare.

\section{Informed Consent}

Informed consent was obtained.

\section{Author Contributions}

MG, YM, AL, and CV were the treating physicians and did the monitoring of the disease. MG wrote the manuscript with the support from ET; and GP supervised the project. All authors discussed the results and contributed to the writing of the final manuscript.

\section{Data Availability}

Any inquiries regarding supporting data availability of this study should be directed to the corresponding author.

\section{References}

1. Zhu N, Zhang D, Wang W, Li X, Yang B, Song J, Zhao X, et al. A novel coronavirus from patients with pneumonia in China, 2019. N Engl J Med. 2020;382(8):727-733.

2. Centers for Disease Control and Prevention. Coronavirus. 2020. https:/www.cdc.gov/coronavirus/2019-ncov/hcp/ pediatric-hcp.html. Accessed on September 07, 2020.

3. Daily report of the Government of Mexico, Covid-19, Mexico. https://coronavirus.gob.mx/datos/; Accessed on September 07, 2020.

4. Riphagen S, Gomez X, Gonzalez-Martinez C, Wilkinson N, Theocharis P. Hyperinflammatory shock in children during COVID-19 pandemic. Lancet. 2020;395(10237):1607-
1608.

5. Verdoni L, Mazza A, Gervasoni A, Martelli L, Ruggeri M, Ciuffreda M, Bonanomi E, et al. An outbreak of severe Kawasaki-like disease at the Italian epicentre of the SARS-CoV-2 epidemic: an observational cohort study. Lancet. 2020;395(10239):1771-1778.

6. Royal College of Paediatrics and Child Health Guidance: paediatric multisystem inflammatory syndrome temporally associated with COVID-19. https://www.rcpch.ac.uk/ sites/default/files/2020-05/COVID-19-Paediatric-multisystem-\%20inflammatory\%20syndrome-20200501.pdf.

7. Centers for Disease Control and Prevention. Emergency preparedness and response: health alert network. Published May 14, 2020. 2020. https://emergency.cdc.gov/ han/2020/han00432.asp. Accessed September 7, 2020.

8. Chiotos K, Bassiri H, Behrens EM, Blatz AM, Chang J, Diorio C, Fitzgerald JC, et al. Multisystem inflammatory syndrome in children during the coronavirus 2019 pandemic: a case series. J Pediatric Infect Dis Soc. 2020;9(3):393-398.

9. Toubiana J, Poirault C, Corsia A, Bajolle F, Fourgeaud J, Angoulvant F, Debray A, et al. Kawasaki-like multisystem inflammatory syndrome in children during the covid-19 pandemic in Paris, France: prospective observational study. BMJ. 2020;369:m2094.

10. Whittaker E, Bamford A, Kenny J, Kaforou M, Jones CE, Shah P, Ramnarayan P, et al. Clinical characteristics of 58 children with a pediatric inflammatory multisystem syndrome temporally associated with SARS-CoV-2. JAMA. 2020;324(3):259-269.

11. Feldstein LR, Rose EB, Horwitz SM, Collins JP, Newhams MM, Son MBF, Newburger JW, et al. Multisystem inflammatory syndrome in U.S. children and adolescents. N Engl J Med. 2020;383(4):334-346.

12. Davies P, Evans C, Kanthimathinathan HK, Lillie J, Brierley J, Waters G, Johnson M, et al. Intensive care admissions of children with paediatric inflammatory multisystem syndrome temporally associated with SARS-CoV-2 (PIMS-TS) in the UK: a multicentre observational study. Lancet Child Adolesc Health. 2020;4(9):669-677.

13. Belhadjer Z, Meot M, Bajolle F, Khraiche D, Legendre A, Abakka S, Auriau J, et al. Acute heart failure in multisystem inflammatory syndrome in children (MIS-C) in the context of global SARS-CoV-2 pandemic. Circulation. 2020.

14. Henderson LA, Canna SW, Schulert GS, Volpi S, Lee PY, Kernan KF, Caricchio R, et al. On the alert for cytokine storm: immunopathology in COVID-19. Arthritis Rheumatol. 2020;72(7):1059-1063.

15. Katzelnick LC, Gresh L, Halloran ME, Mercado JC, Kuan G, Gordon A, Balmaseda A, et al. Antibody-dependent enhancement of severe dengue disease in humans. Science. 2017;358(6365):929-932. 